\title{
22
}

\section{An Approach to Modeling Real-Time Control of Dynamic and Static Radial and Sluice Gates within EXTRAN}

\author{
William James and Brett Charles Young
}

This chapter presents an approach to analysing combined sewer systems with dynamic sluice and radial gates for real-time flow control. New code was developed for radial gates and integrated into version 4 of the Extended Transport(EXTRAN) module of the Stormwater ManagementModel(SWMM4). The new program is called RGEXTRAN and is suitable for handling unsteady flow conditions occurring in real sewer systems with radial and regular sluice gates. The program also makes provision for dynamic control of the radial gates (or other gate types) based on flow conditions at remote locations in the sewer system. The radial gate code has been verified under a wide range of flow conditions, and found to produce good results for most conditions. The code can be used for dynamic or static sluice gates as well as radial gates by using the "constant discharge coefficient" option. RGEXTRAN is subject to the numerical stability concerns associated with the explicit scheme used in the EXTRAN program. This chapter covers the background and describes the verification of the new code.

\subsection{Introduction}

Combined sewers transport both sanitary sewerage and stormwater in the same pipe. During wet weather stormwater flows may exceed the conveyance capacity of the system and excess flow spilled directly (untreated) to the receiving waters, as a combined sewer overflow (CSO). CSOs have been

James, W. and B.C. Young. 2001. "An Approach to Modeling Real-Time Control of Dynamic and Static Radial and Sluice Gates within EXTRAN." Journal of Water Management Modeling R207-22. doi: 10.14796/JWMM.R207-22.

(C) CHI 2001 www.chijournal.org ISSN: 2292-6062 (Formerly in Models and applications to Urban Water Systems. ISBN: 0-9683681-4-X) 
recognized as a significant source of pollution to aquatic environments, and regulations to reduce CSOs are being enforced in many cities in North America and worldwide. While many models exist for analyzing combined sewer systems, few models account for all features of real systems (e.g., diversion structures), and real hydraulic conditions (e.g., backwater, reverse flow and surcharge). Many installed CSO systems incorporate dynamic radial gates, where operation of the gates is controlled in real time by water levels at remote locations in the system. Often these gates are the principal regulating structures, and have a significant effect on hydraulic conditions in the sewers. In the present study, new code was developed for radial gates and the code was verified using simple sewer networks and test hydrographs. Verification tests were done over a wide range of flow conditions to test the code for all possible conditions that may occur in real sewer systems. The following criteria were used to evaluate the performance of the new program: (i) that stability be maintained in the simulation model, (ii) that continuity be maintained in the model, and (iii) that the resulting depth-discharge (Y-O) relationship for radial gates be reasonable and consistent with results reported in the literature for steady-state conditions. The code can also be used for dynamic or static sluice gates as well as radial gates, by using the "constant discharge coefficient" option. This chapter describes the background development and verification of the radial gate code.

\subsection{Background Review of Radial Gates}

Radial, or tainter, gates are commonly used as flow-control structures for a wide range of hydraulic applications. They are found in canal systems, on reservoir spillway crests, in water supply systems, and in sewers of various sizes. In sewer systems, such gates may also be used to utilize available storage within the sewer (Book, 1980).

They have the advantage that the hydrodynamic forces on the seal are relatively small, the pinion can be built relatively friction-free, and by manipulating the height of the pinion above the floor, hydrostatic forces on the gate can be used to either open or close the gate without external energy.

Discharge characteristics for radial gates have traditionally been determined by special hydraulic model studies. Discharge characteristics are dependent on the specific geometry of the gate as well as the angle and design of the gate lip, and are thus unique to each gate structure. However, by presenting design charts for gate discharge coefficients in a dimensionless form, experimental results may be extended to other radial gates of similar design (Metzler 1948, Toch 1953; Buyalski 1983). 
Most theoretical and experimental studies are limited to radial gates in prismatic channels, on horizontal surfaces and under open channel flow conditions. Very little information on radial gate characteristics under surcharge conditions is available. The U.S. Army Corps of Engineers Hydraulic Design Chart 320-3 (Buyalski, 1983) is available for estimating discharge coefficients for radial gates in conduits. Unfortunately, their results are presented in design charts that are not suitable for use in a computer model. Variables used in this review are defined in Figure 22.1.

\subsubsection{Features of Radial Gates}

A radial gate consists of a curved face, usually smooth, supported by braced radial arms. When a hydrostatic load is applied to the gate face, the resultant force acting on the gate passes directly through the shaft axis, and thus the hoist mechanism lifts only the gate mass, which can be balanced by hydrostatic forces. This allows for the design of a smaller hoist mechanism (Chadwick and Morfett, 1986). Furthermore, these gates do not require a complicated groove system to prevent leakage while transmitting the hydraulic thrust to the side walls, but instead have rubber seals in direct contact with the side walls (Bos, 1978).

Curvature of the radial gate face provides a more hydraulically-efficient shape than other gate types. The discharge coefficient used in the flow equation is dependent on the contraction of the jet below the gate. As the gate is raised or lowered, the angle of the gate lip, $\theta$ (Figure 22.1) changes and consequently affects the contraction of the jet. This needs to be accounted for when calculating accurate values for discharge coefficients.

As with other underflow gates, the radial gate discharge may be either free or submerged, as shown in Figure 22.1. For free flow conditions the gate discharge is dependent on the upstream conditions only, and the downstream conditions have no effect on the gate discharge. However, submerged (or drowned) discharge is controlled by both upstream and downstream conditions, and thus downstream water levels need to be considered in the flow computations. In this case, the effective head on the gate is the difference between upstream and downstream water levels. For the same upstream water level the effective head on the gate is decreased as the gate becomes submerged. The exact depth downstream of the gate is difficult to calculate or measure accurately in the field or laboratory, due to the high turbulence in this region. Therefore, it is usual to estimate this depth from known values of the gate opening and conditions further downstream from the gate structure, where it is possible to obtain a more accurate measure of the flow depth (Bos, 1978; Gill, 1982). 


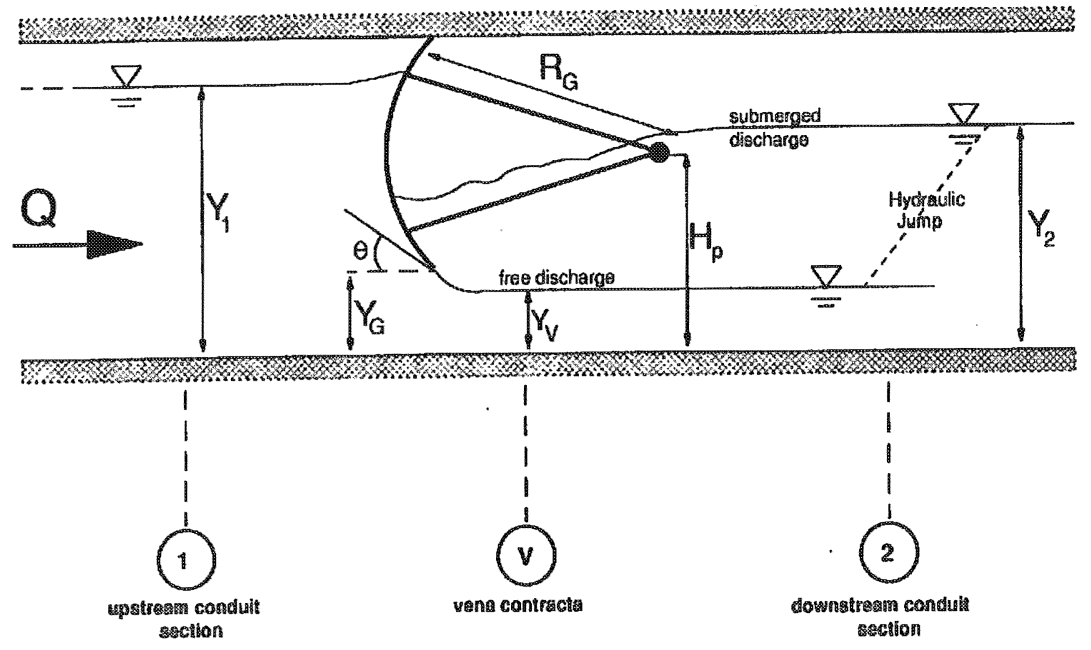

Figure 22.1 Radial gate notation.

Under unsteady flow conditions, the flow under the gate may shift from one condition to another, further complicating the discharge calculation. In sewer systems, this problem becomes more pronounced as conditions change rapidly and the conduit may surcharge or be susceptible to severe backwater and possible reverse flows. Thus, the difficulty of applying the steady-state discharge equations to sewer networks is apparent.

\subsection{Background Theory}

The theoretical analysis of radial gate hydraulics presented here uses the steadystate equations of continuity, energy, and momentum for open channel flow. Chow (1959) expressed the discharge through an underflow gate:

$$
Q=C_{d} L_{G} Y_{G}\left[2 g\left(Y_{1}+V_{1}^{2} / 2 g\right)\right]^{0.5}
$$

where:

$$
\begin{aligned}
C_{d} & =\text { discharge coefficient, } \\
L_{G} & =\text { gate width in } \mathrm{m}[\mathrm{ft}] \\
Y_{G} & =\text { vertical gate opening in } \mathrm{m}(\mathrm{ft}] \\
Y_{1} & =\text { depth upstream of the gate in } \mathrm{m}[\mathrm{ft}], \text { and } \\
V_{1} & =\text { flow velocity upstream of gate in } \mathrm{m} \cdot \mathrm{s}^{-1}\left[\mathrm{ft} \cdot \mathrm{s}^{-1}\right] .
\end{aligned}
$$


The velocity-head term $V_{1}^{2} / 2 g$ is often omitted from the above equation and its effect accounted for in the value of the discharge coefficient (Chow, 1959). The simplified form of the discharge equation then becomes:

$$
Q=C_{d} L_{G} Y_{G}\left[2_{g} Y_{l}\right]^{0.5}
$$

For submerged discharge, the term $Y_{1}$ should be replaced by the effective head (the difference between the water depths immediately upstream and downstream of gate). Often, however, Equation 22.2 is also used in the submerged discharge calculations and the discharge coefficient becomes dependent on both the upstream and downstream water depths, $Y_{1}$ and $Y_{2}$ respectively. Note that $Y_{1}$ and $Y_{2}$ are considered to be sufficiently far from the gate to be not influenced by local turbulence.

When deriving the equations for radial gate discharge the assumptions of constant velocity along the free streamline and constant hydrostatic pressure across the effluent jet are usually made for both free and submerged flow. However, Toch (1953) cautions that the application of hydrodynamic theory is only theoretically correct for free jet efflux where the tailwater does not interfere with the jet emanating under the gate.

A theoretical analysis, accounting for the non-uniform velocity distribution of the effluent jet is presented by Gill (1982). In his paper, Gill introduces a correction factor $\alpha_{f}$ to account for non-uniform velocity. For uniform velocity distribution, the value of $\alpha_{f}$ is unity. Franke and Valentin (1969) present a method for determining the discharge under a sluice gate as a function of the depth upstream and the pressure distribution on the channel floor. This method is useful in cases of variable tailwater depth, as the downstream conditions are not considered in the calculations. Isaacs (1977) also developed a numerical method, based on finite element techniques, for analyzing flow under sluice gates of arbitrary geometry. The theoretical analysis that follows has been summarized from selected sources: (Toch, 1953; Chow, 1959; Henderson, 1966; Larock, 1970; Bos, 1978; Gill, 1982; Ranntz, 1982; Buyalski, 1983).

\subsubsection{Free Discharge}

The discharge of the gate is defined as being free when the jet of water under the gate is exposed to the atmosphere and the gate lip is not submerged below the tailwater depth. Flow immediately downstream of the gate is supercritical and a hydraulic jump is formed some distance downstream of the gate, if the channel has a hydraulically mild slope.

Application of the continuity and energy equations between [1] and [V] in Figure 22.1 yields: 


$$
Q=L_{G} Y_{1} Y_{V}\left[2 g /\left(Y_{1}+Y_{V}\right)\right]^{0.5}
$$

The depth at the vena contracta, $Y_{V}$ is:

$$
Y_{V}=C Y_{G}
$$

where $C=$ contraction coefficient.

Thus:

$$
Q=C_{d} L_{G} Y_{G}\left[2 g Y_{1}\right]^{0.5}
$$

The discharge coefficient $C_{d}$ is given by:

$$
C_{d}=C\left[Y_{1} /\left(Y_{1}+C Y_{G}\right)\right]^{0.5}
$$

For preliminary design purposes, Henderson (1966), proposed the following equation for calculating the contraction coefficient:

$$
C=1-0.75\left(\theta / 90^{\circ}\right)+0.36\left(\theta / 90^{\circ}\right)^{2}
$$

where $\theta=$ lip angle in degrees.

This relationship is shown graphically in Figure 22.2. Inspection of this graph shows that the lip angle of the gate significantly affects the coefficient of contraction and thus the value of $C_{d}$. For variation of the lip angle from $0^{\circ}$ to $90^{\circ}, C$ decreases from 1.0 to approximately 0.61 . Normally the gate width $L_{G}$ and the gate opening $Y_{G}$ as well as the depth $Y_{1}$ are known, and are used to determine the discharge coefficient. The determination of the discharge coefficient is thus the primary concern in the calculation of the radial gate discharge under steady-state conditions.

As mentioned, Gill (1982) includes a correction factor $\alpha_{f}$ to account for the non-uniform velocity distribution, and replaces $C_{d}$ by $C_{r}$ where :

$$
C_{r}=C /\left[\alpha_{f}+\left(C Y_{G} / Y_{\nu}\right)^{2}\right]^{0.5}
$$

\subsubsection{Submerged Discharge}

Submerged discharge occurs when the tailwater drowns the lower lip of the raised gate (Ranntz, 1982). Under these conditions the downstream depth is greater than the sequent depth at which a hydraulic jump would form (for a hydraulically mild slope). In this condition, the gate discharge is influenced by both the downstream and the upstream conditions. The submerged discharge 


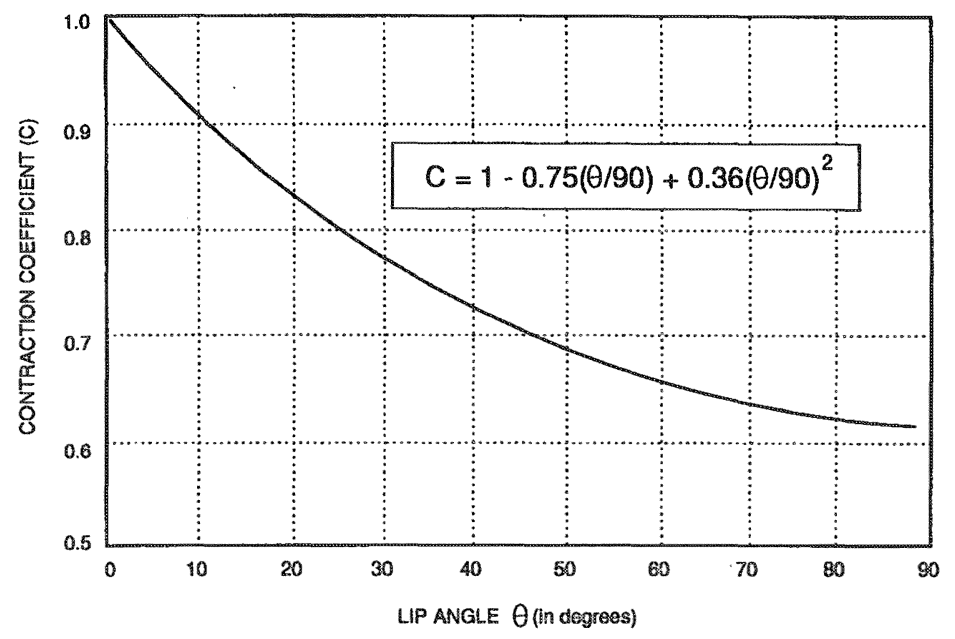

Figure 22.2 Contraction coefficient.

can be approximated theoretically by applying the continuity and momentum equations together with a modification of the Bernoulli equation (Toch, 1953). In the analysis of submerged discharge, the assumption is made that the hydrostatic pressure distribution also prevails in the turbulent region immediately downstream of the gate. It is also assumed that all head losses occur in the region between sections [V] and [2] in Figure 22.1, and that there is no loss of energy between sections [1] and [V] (Toch, 1953).

Application of the energy equation between sections [1] and [V] yields:

$$
-Y_{I}+Q^{2} /\left(2 g L_{G}{ }^{2} Y_{I}^{2}\right)=Y_{s}+Q^{2} /\left(2 g L_{G} 2 Y_{v}^{2}\right)
$$

where $Y_{s}=$ depth of submerged flow in $\mathrm{m}$ [ft].

Using the momentum equation between section [V] and [2] leads to:

$$
Y_{s}^{2} / 2+Q^{2} /\left(2 g L_{G}{ }^{2} Y_{v}\right)=Y_{2}^{2} / 2+Q^{2} /\left(2 g L_{G}{ }^{2} Y_{2}\right)
$$

where: $\quad Y_{2}=$ depth of downstream flow in $\mathrm{m}[\mathrm{ft}]$, and $Y_{v}$ is given by the earlier equation.

$Y_{s}$ is difficult to measure because of turbulence and has to be estimated from $Y_{2}$, which is much easier to measure (Gill, 1982). Equations 22.9 and 22.10 can then be solved simultaneously for the discharge $Q$. 
Generally, however, as an alternative, Equation 22.2 is used to calculate submerged discharge (Gill, 1982), with the discharge coefficient modified to account for submergence. Thus:

$$
Q_{s}=C_{d s} L_{G} Y_{G}\left[2 g Y_{1}\right]^{0.5}
$$

where $C_{d s}=$ discharge coefficient for submerged flow.

Expressions for submerged discharge coefficients are given by Toch (1953), Gill (1983) and the U.S. Bureau of Reclamation (Buyalski, 1983). Toch (1953) presents an expression for determining discharge coefficients for submerged efflux based on a head-loss parameter. Unfortunately his results were based on a limited range of gate geometries and are not suitable for use in computer programs. Gill (1983) gives the following explicit expression for $C_{d s}$ :

$$
C_{d s}=C\left[\beta(\beta-\varphi) /\left(\beta^{2}-C^{2}\right)\right]^{0.5}
$$

where

$$
\begin{aligned}
& \beta=\mathrm{Y}_{1} / \mathrm{Y}_{\mathrm{G}} \\
& \varphi=\mathrm{Ys} / \mathrm{Y}_{\mathrm{G}} \\
& C \text { is given by Equation } 22.7
\end{aligned}
$$

For both free and submerged flow, discharge coefficients for radial gates are usually determined from special experimental and hydraulic model studies. It has become customary to present these results in a dimensionless form similar to Figure 22.3. The discharge coefficient is a function of the geometry of the radial gate, often expressed by the dimensionless ratios of: the pinion height $H_{p}$ to the gate arm radius $R_{G}$; the upstream depth $Y_{i}$; the vertical gate opening $Y_{G}$, and the contraction of the jet under the gate. As described, the jet contraction is defined by the gate lip angle $\theta$ which may be expressed in terms of $H_{p}, Y_{G}$ and $R_{G}$. For submerged discharge, the downstream depth $\mathrm{Y}_{2}$ in the dimensionless form $Y_{2} / R_{G}$ is also considered. Some authors use the pinion height $H_{p}$ in place of the arm radius as the base reference for the dimensionless plots. Figure 22.3 shows the discharge coefficient for submerged flow has a much wider range than that for free discharge. For low $Y_{1} / Y_{G}$ ratios, $C_{d}$ is almost indeterminate. Even at higher ratios, the discharge coefficient for submerged flow is very sensitive to depth changes and varies significantly with small changes in depth. Thus $C_{d}$ for submerged flow is much more sensitive to errors in measured quantities, than $C_{d}$ is for free flow. The submergence of the gate therefore makes the gate unsuitable for accurate flow control and measurement (Bos, 1978). Under unsteady flow conditions, it becomes much more difficult to accurately compute the submerged discharge coefficients. 


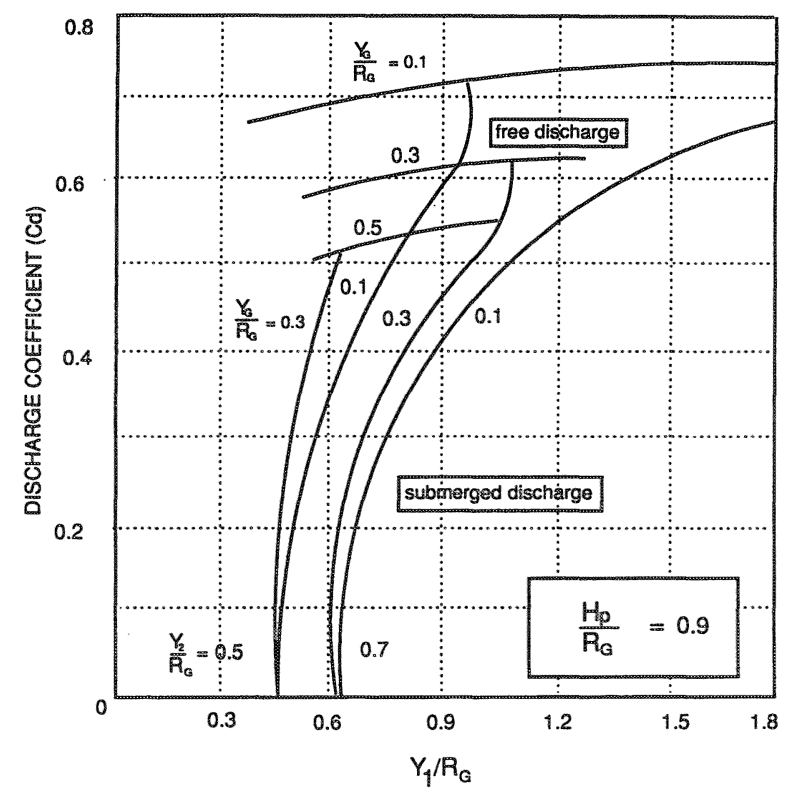

Figure 22.3 Discharge coefficient.

Metzler (1948) was the first to use a non-dimensional approach to present the discharge coefficients for radial gates, and expressed the discharge coefficient as a function of three dimensionless ratios (see Figure 22.1):

$$
C_{d}=f n\left(Y_{G} / H_{p}, Y_{1} / H_{p}, Y_{2} / H_{p}\right)
$$

Because of the dimensionless nature of Figure 22.3, the curves can be applied to other radial gates of similar geometric proportions. For free discharge, Metzler found that the coefficient of discharge decreases as the gate opening increases. However, the discharge coefficient increases with increased gate opening for submerged flow. As the flow area and discharge both increase with gate opening, this discrepancy is explained by the relative rate of the increase between the area and the discharge: for free flow, the area increases at a faster rate than the discharge; and for submerged flow, the reverse is true.

\subsubsection{The U.S. Bureau of Reclamation (BUREC)}

BUREC algorithms compute the discharge coefficients with an average error of $+0.36 \%$ and a standard deviation of $4.9 \%$, when compared to laboratory test results. Field verification tests were also undertaken in which the algorithms 
were used to compute the discharge for thirteen canal radial gate structures designed by BUREC. The average error of the discharge calculated by the algorithms was $+0.7 \%$, with a standard deviation of $4.9 \%$. The design of the radial gate lip seal was found to have a significant effect on the determination of the discharge coefficient. Use of the different designs in this study indicated that the discharge coefficient could vary from -10 to $+12 \%$, when other gate seal designs were compared to the sharp edged design. Use of the correction algorithms resulted in average errors of approximately $0 \%$, in most tests done byBUREC.

The high degree of accuracy of the algorithms is limited to radial gates having the same characteristics as those designed and used by BUREC. These limitations may be expressed in terms of the following two ratios: the gate arm radius to the pinion height $R_{G} / H_{p}$ and the maximum flow depth to the pinion height $Y_{I} / H_{p}$. The ratio $R_{G} / H_{p}$ should be in the approximate range of 1.2 to 1.7. The water depth to pinion height ratio should not exceed 1.6. This is a reasonable range when considering radial gates found in sewer systems. However, caution should be exercised when applying the algorithms to radial gates with dimensions outside these limits. Additional constraints are that the gate faceplate be smooth, and the canal invert should be horizontal (or nearly horizontal) from the start of the pier upstream to the end of the pier downstream; or in the immediate area upstream and downstream of the gate if no piers are present.

\subsubsection{Unsteady Flow}

Book (1980) presents a computer program capable of simulating sluice gates using a constant discharge coefficient. In an explicit finite difference scheme, it is possible to apply the steady-state equations within a time-step, as is done with orifices and weirs in the EXTRAN program (Roesner et al., 1989). However, the time step chosen should be sufficiently small to ensure that there is little change in upstream and downstream water levels during the time-step (Hamilton, 1985):

$$
Y_{1}^{t}-Y_{1}^{t+1}<d Y
$$

where $d Y=$ tolerance level.

For large canal systems, the time-step can usually be of the order of a few minutes (Hamilton, 1985). However, for sewers, where more rapid flow changes occur, the time-step should be smaller, often 10-60 s. 


\subsection{New Coding for Dynamic Radial Gates}

In this project, new code was written for simulating radial gate control structures in sewer systems. The program computes the discharge through radial gates under all flow conditions, and also simulates dynamic controls on the gate, i.e. where the operation of the gate is controlled by hydraulic conditions elsewhere in the sewer system.

\subsubsection{Conceptual Representation of Radial Gates in RGEXTRAN}

Radial gates are handled in RGEXTRAN the same way as are the flow diversion structures in EXTRAN. Thus, RGEXTRAN considers the radial gate as a link, which transfers flow between two nodes. The dependent variable for the radial gate link is the discharge, which is calculated explicitly from known values of the upstream and downstream depth. No length is specified for this link, and the particular geometry of the gate and the regulating chamber (intake and outflow sections in Figure 22.4) is accounted for in the radial gate computations. This approach allows the steady-state radial gate equations to be solved directly at each half time-step. The conceptual representation of a radial gate link as used in RGEXTRAN is shown in Figure 22.5.

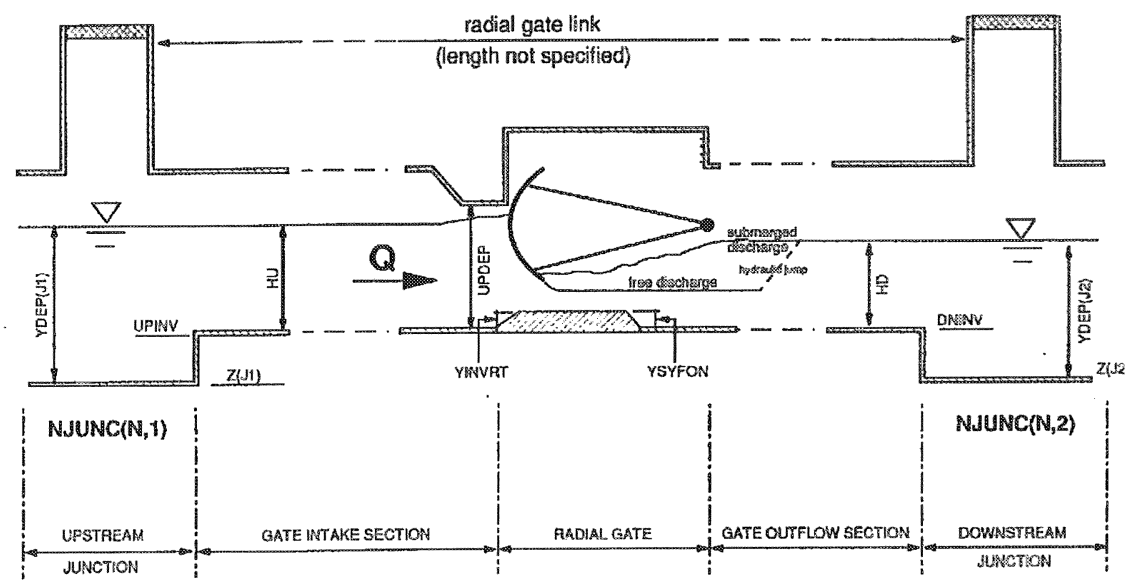

Figure 22.4 Radial gate parameters. 


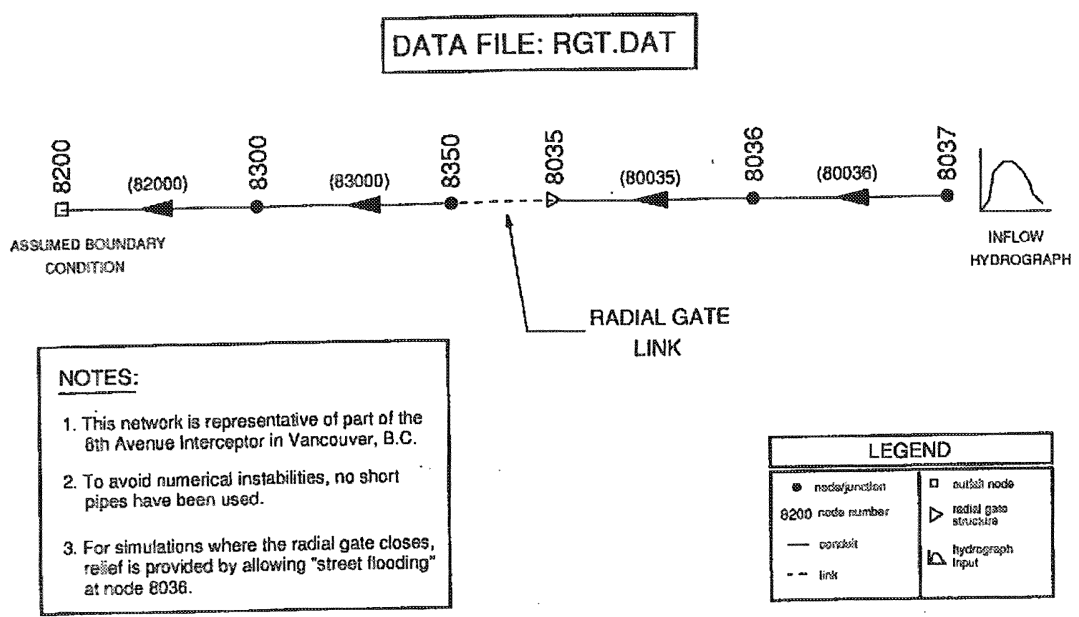

Figure 22.5 Radial gate link schematic.

\subsubsection{Input Data for Radial Gates}

The input data for radial gates in RGEXTRAN is similar to that for weirs and orifices in the original EXTRAN program. However, as a result of the complex geometry and hydraulics of these structures, the new input data is more complex. The required input data includes variables describing the gate geometry, structural details and hydraulic assumptions for the gate chamber, iteration parameters, and data describing the gate operating characteristics. Figure 22.5 was used for verification of the new radial gate code. The program prints an echo of the radial gate input data to the results file, and checks the input data for errors. Appropriate warning messages are printed to facilitate corrections. The new code is suitable for both SI and U.S. customary units. All verification tests were done using U.S. customary units as these are the standard units for the available data.

\subsubsection{Dynamic Control of Gate}

In order to allow dynamic control of the radial gates in the model, it is necessary that the gate opening vary during the simulation based on conditions elsewhere in the system. Such dynamic controls allow for improved sewer system operation by making better use of the available insystem storage and conveyance capacity, and protect the downstream sewers from surcharge and flooding. 
In RGEXTRAN, the vertical radial gate opening is controlled by water levels at any junction in the network specified by the user. The computed gate opening is assumed to be constant for the duration of a time step (typically 10 to 30 seconds). The program accounts for two means of specifying the dynamic gate operation, i.e. time-controlled or depth-controlled.

For time-controlled gates, the gate remains at a constant opening until triggered by the water level at the specified location to begin closing. The closure cycle of the gate continues at the rate specified by the user until fully closed or until the gate is triggered to reopen. Once fully closed, the gate remains this way until the water depth at the controlling node signals the gate to begin reopening. The rate of opening is also defined by the user. The gate then continues to open to the maximum allowable opening specified, unless the closure cycle is again triggered. Figure 22.6 gives an example discharge and depth hydrograph showing the effect of the dynamic radial gate operation. This plot was generated using the network in Figure 22.5.
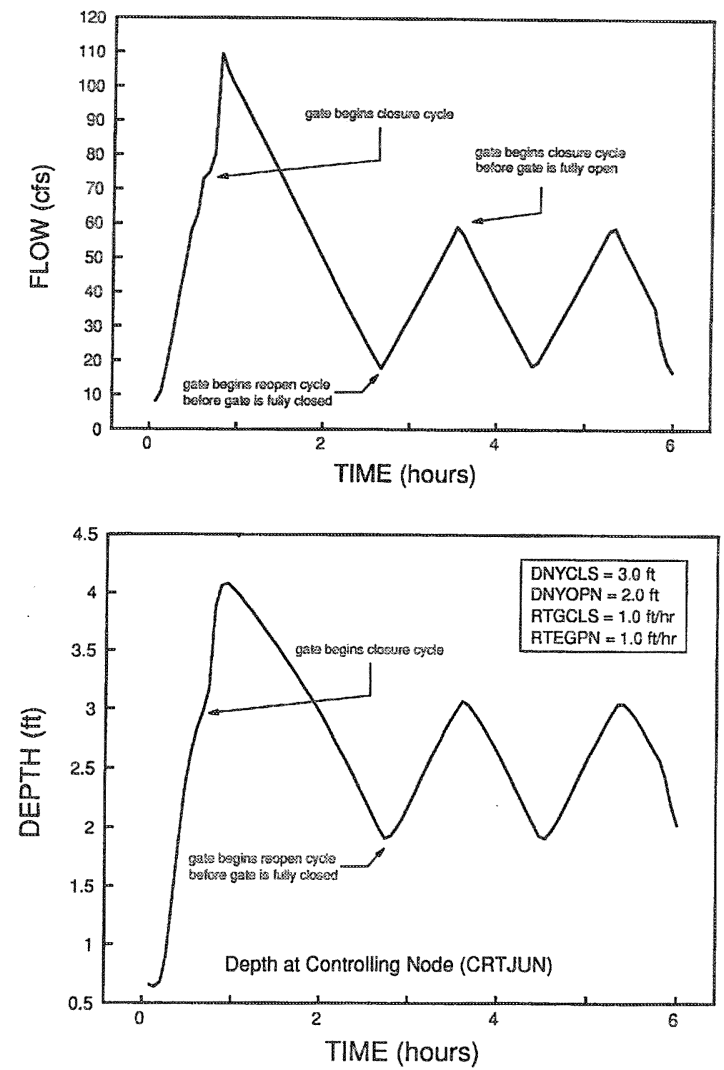

Figure 22.6 Computed hydrographs, remote control. 
For depth-controlled gates, the vertical gate opening is assumed to have a direct, unique relationship to the depth at the controlling node. The input for this type of control is a user-defined table of depth versus vertical gate opening. A table look-up routine, using linear interpolation, computes the vertical gate opening at each time step.

It is possible for both time- and depth-controlled gates that, under certain conditions, the gate may begin to "hunt" in the model, as it is successively opened and closed by changes in water levels at the controlling node. This may occur in the real system for certain conditions and operating characteristics. However, artificial hunting may also be caused by instabilities and inaccuracies in the program computations. Steps for eliminating hydraulic and numerical instabilities, and thus artificial hunting, are discussed below. Care must also be taken when specifying the radial gate operating characteristics to ensure that the input variables do not cause artificial hunting in the model.

\subsubsection{Code Verification}

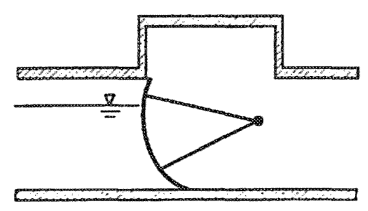

1. Gate Closed

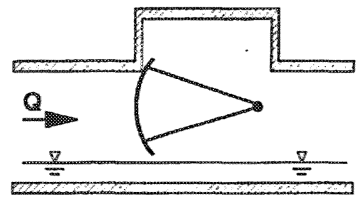

3. Unrestricted Flow

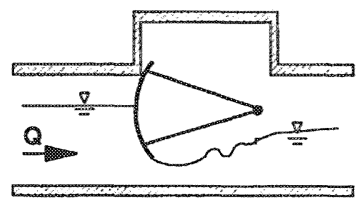

5. Transition

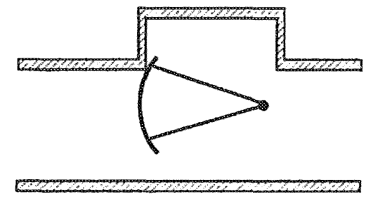

2. No Flow

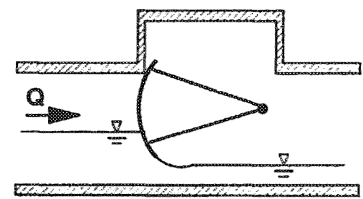

4. Free Discharge

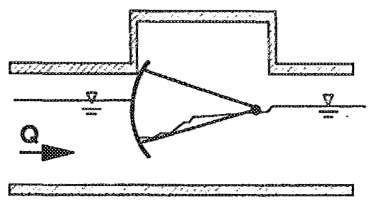

6. Submerged Discharge

Figure 22.7 Gate flow conditions. 
In order that the program run successfully under unsteady storm flow conditions, the possible hydraulic conditions that are considered and accounted for in the program are illustrated schematically in Figures 22.7 and 22.8. They form the basis for the code verification tests.

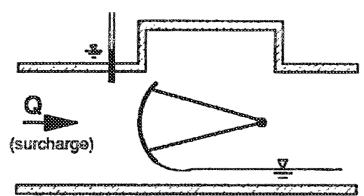

7. Upstream Surcharge

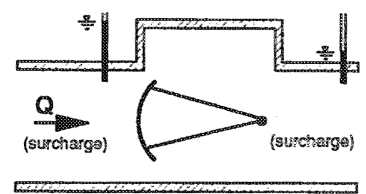

8. Total Surcharge

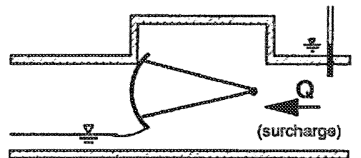

9. Surcharge Reversal

\section{SURCHARGE CONDITIONS}

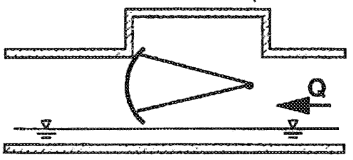

10. Unrestricted Flow

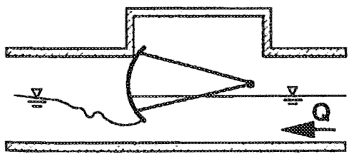

12. Transition

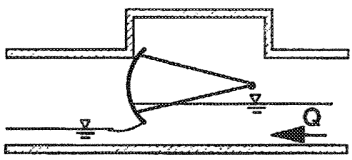

11. Free Discharge

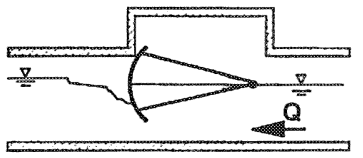

13. Submerged Discharge

FLOW REVERSAL CONDITIONS

Figure 22.8 Gate surcharge and reversal conditions.

\section{Zero Discharge (Conditions 1 and 2)}

Zero discharge occurs when there is no flow in the conduit upstream of the gate or when the gate is in the fully closed position. When the gate is fully closed, the upstream flow is reversed and diverted at a junction upstream of the radial gate.

\section{Unrestricted Discharge (Condition 3)}

This condition occurs when the flow depth is less than the vertical gate opening. For these conditions, the discharge for the radial gate link is computed by Manning's equation. The value of the friction factor, or Manning's $n$, for the 
radial gate link is defined by the user to account for any localized energy losses and may be used as a calibration parameter. An example hydrograph for unrestricted flow, as computed by RGEXTRAN, is given in Figure 22.9. It is important to note that these conditions are unlikely to occur for significant periods in a real sewer system, except under dry weather flow conditions, which are not important in most model applications.
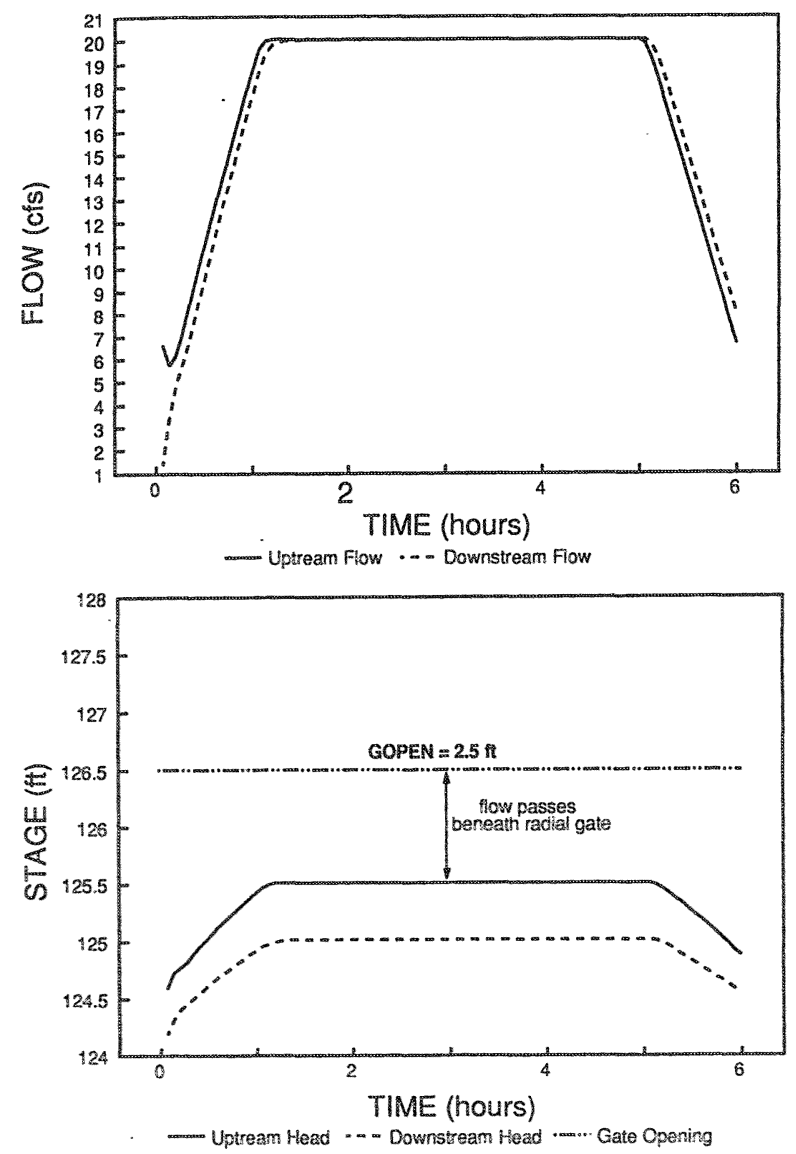

Figure 22.9 Computed hydrographs, unrestricted flow.

\section{Regular Discharge (Conditions 4, 5, and 6)}

Regular conditions in this context refer to the free surface flow conditions shown in Figure 22.7. These conditions are the most likely to occur during regular operating conditions. In general, under transient conditions, the discharge condition of the radial gate (i.e. free or submerged) cannot be 
computed and may change during the simulation. The radial gate code accounts for this, and determines which condition is appropriate. The code also accounts for the presence of a gate sill, and any head losses which may occur in the gate intake and outflow sections (Figure 22.4). The discharge calculations for conditions 4,5 , and 6 . begin by assuming the gate discharge to be in the free flow condition. An iterative procedure is used to determine the depth immediately upstream of the gate. This is done by assuming an initial estimate of the discharge coefficient and applying the energy equation between the upstream junction, where the depth is known, and the upstream end of radial gate section (see Figure 22.4), accounting for the effects of the gate sill and any head losses in the intake section. This computed depth is then used in the BUREC free discharge algorithms to compute an accurate discharge coefficient, and thus the radial gate discharge. The Newton method is used in the iterative procedure to achieve convergence based on the user defined tolerances. The program then computes the discharge assuming the radial gate to be submerged. The procedure for these computations is similar to that described above. However, in this case the discharge is influenced by downstream conditions, and the energy equation is applied downstream of the gate (between the gate section and the downstream junction in Figure 22.4), as well as upstream of the gate. Warning messages are printed for both the free and submerged discharge calculation if the iterative routines fail to converge, or if continuity is not satisfied. The program keeps account of the occurrence of these errors and is terminated if the number of errors in a simulation exceed 100 . The discharge coefficient calculated using the submerged radial gate algorithms is very sensitive to changes in the flow depth and may cause instabilities in the model results under certain conditions. Once these computations are complete, a test is performed to determine the flow condition. This test consists of comparing the momentum and hydrostatic forces upstream and downstream of the gate for the computed values of the free and submerged discharge. If the upstream forces are greater for both conditions, the flow condition is assumed to be free. If the downstream forces are greater in both cases, the gate discharge is assumed to be submerged. If the results of the two cases differ, the flow is assumed to be in a transition range between free and submerged discharge. This technique was adapted from the program presented by BUREC (Buyalski, 1983).

\section{Surcharged Flow (Conditions 7, 8, and 9)}

Surcharge of the gate is defined as occurring when the upstream head exceeds the height of the intake structure housing the gate; i.e. the upstream approach conduit is flowing full and the free water surface is no longer in contact with the radial gate face. When surcharge is detected, the program uses a constant 
discharge coefficient defined by the user, and the generalized orifice equation to compute the gate discharge. Code verification and testing has shown this approach to be successful, and the results of a typical verification run is given in Figure 22.10. If model instabilities are encountered under surcharge conditions, the radial gate should be replaced by an equivalent pipe.
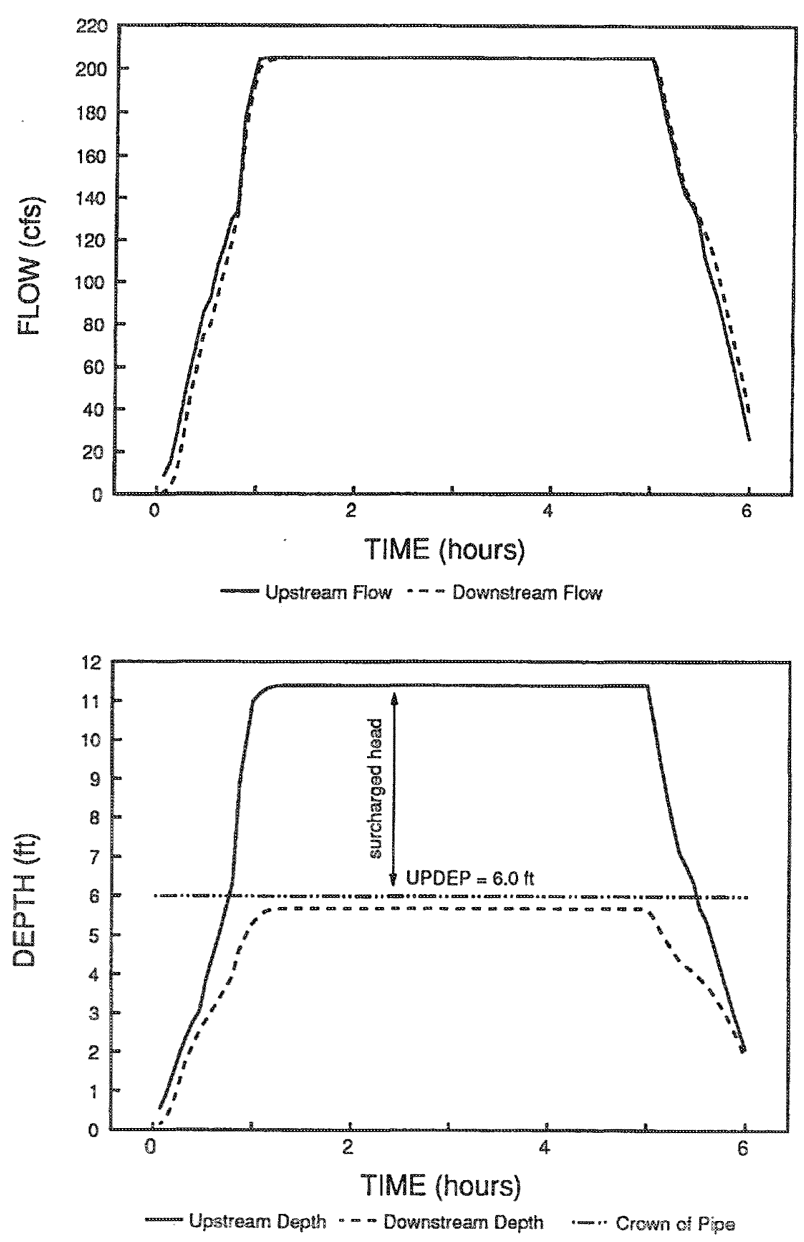

Figure 22.10 Computed hydrographs, surcharged flow.

Flow Reversal (Conditions 10 to 13)

An important feature of a dynamic wave routing model is the ability to simulate flow reversal in the sewer system. Although it is unlikely that flow reversal will occur across a control gate in most real applications, it is important that the code 
account for these conditions, even if the results are only approximate. The program detects flow reversal when the downstream stage exceeds the upstream stage. A tolerance level is built into the code to prevent artificial "hunting" between positive and reverse flow. The program computes the reverse discharge under the gate using the generalized orifice equation and a user specified coefficient. It is assumed that the reverse gate discharge is always in the submerged flow condition, and $Y$ is replaced by the head difference across the gate. The submerged flow assumption implies that the momentum of the reverse jet is insufficient to cause a hydraulic jump on the other side of the gate, which is a reasonable assumption under almost all conditions. The occurrence of reverse flow conditions under control gates is rare in a real sewer system. It would be usual practice to design the gate to close under the presence of high downstream water levels in order to avoid overloading the system, and allow the excess flow to be diverted upstream of the gate. The approach described is suitable for estimating the reverse flow and allowing the simulation to proceed under these conditions.

\subsubsection{Stability}

During the code verification process, it was found that under certain unsteady flow conditions the BUREC algorithms for submerged flow caused unstable results in the model. These hydraulic instabilities only occur for the submerged discharge condition, and are especially noticeable during periods of steeply rising or falling inflow. The cause of these instabilities becomes apparent when considering the sensitivity of the submerged discharge calculations to small changes in flow depth, both upstream and downstream of the gate, as shown in Figure 22.11. This effect is further illustrated by Figure 22.3, which shows a typical dimensionless plot for radial gate discharge coefficients. Examination of Figure 22.3 shows the value of submerged discharge coefficient to be very sensitive to changes in depth, and for low upstream depths $\left(Y_{l}\right)$ the value of $C_{d}$ for submerged discharge is almost indeterminate. When using these algorithms in RGEXTRAN under unsteady storm conditions, the change in flow depth from one time step to the next may result in a relatively large increase in the computed gate discharge. In some cases, the computed gate discharge may exceed the available flow in the upstream conduit resulting in a localized continuity error at the gate. The program attempts to correct this continuity error at the next time-step by modifying the depth, which in turn affects the computed gate discharge. Under certain conditions, this cycle may repeat itself for a significant period resulting in oscillations of the computed results (hydraulic instabilities). 

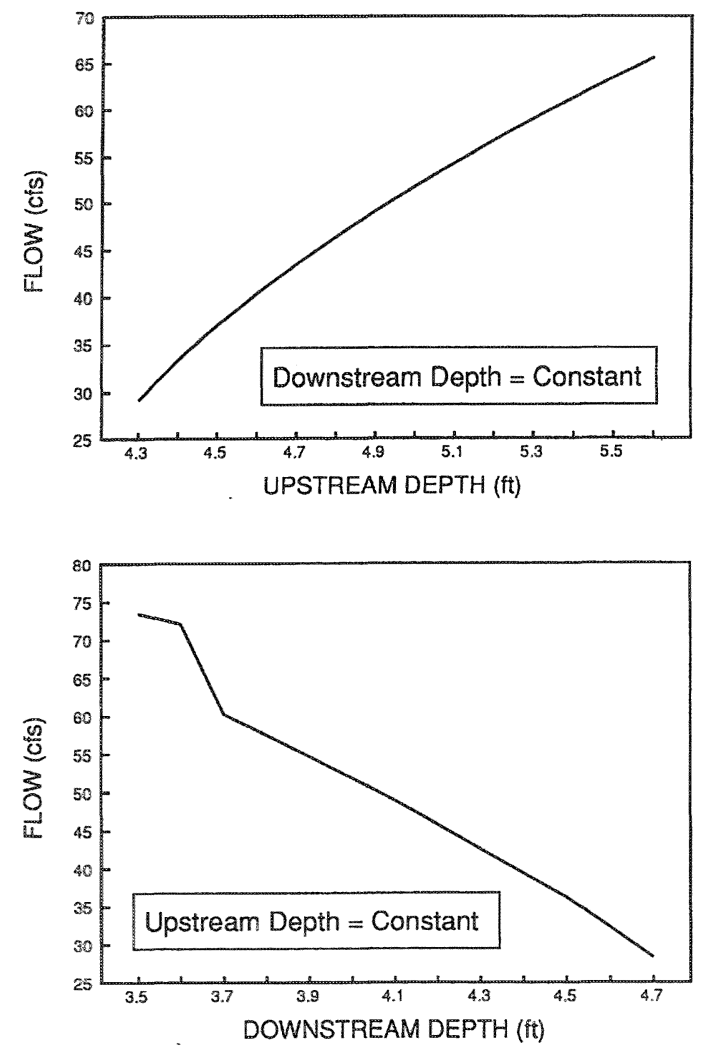

Figure 22.11 Computed rating curves, submerged flow.

These hydraulic instabilities may be alleviated by using a constant discharge coefficient or by replacing the radial gate link by a hydraulically equivalent pipe. The substitution of an equivalent pipe for a diversion structure is a technique used in EXTRAN to overcome stability concerns. This option has been included in RGEXTRAN, and the radial gate will automatically be converted to an equivalent pipe if the parameter DISCND is set to zero. The conversion to an equivalent pipe is achieved by equating the gate equation and the Manning pipe flow equation (Roesner et al., 1989). The cross sectional area of the equivalent pipe is rectangular and equal to the area of the gate opening. For gates with dynamic controls, the equivalent pipe dimensions and roughness parameter is recomputed at each time-step to account for the changes in gate opening. Use of an equivalent pipe for submerged discharge conditions produces more stable results than the BUREC algorithms, and should be used 
in cases where these algorithms have been found to be unsuitable. Guidelines for using this option are given in Table 22.1 (see section 22.5.3). Use of this method is justified by the need to maintain stability in the model, and the consistency with the handling of other diversion structures in EXTRAN. The results in Figure 22.12 illustrate the difference between hydraulic instabilities, resulting from use of the BUREC submerged discharge algorithms, and numerical instabilities caused by the use of a short pipe length, which violated the stability criterion.
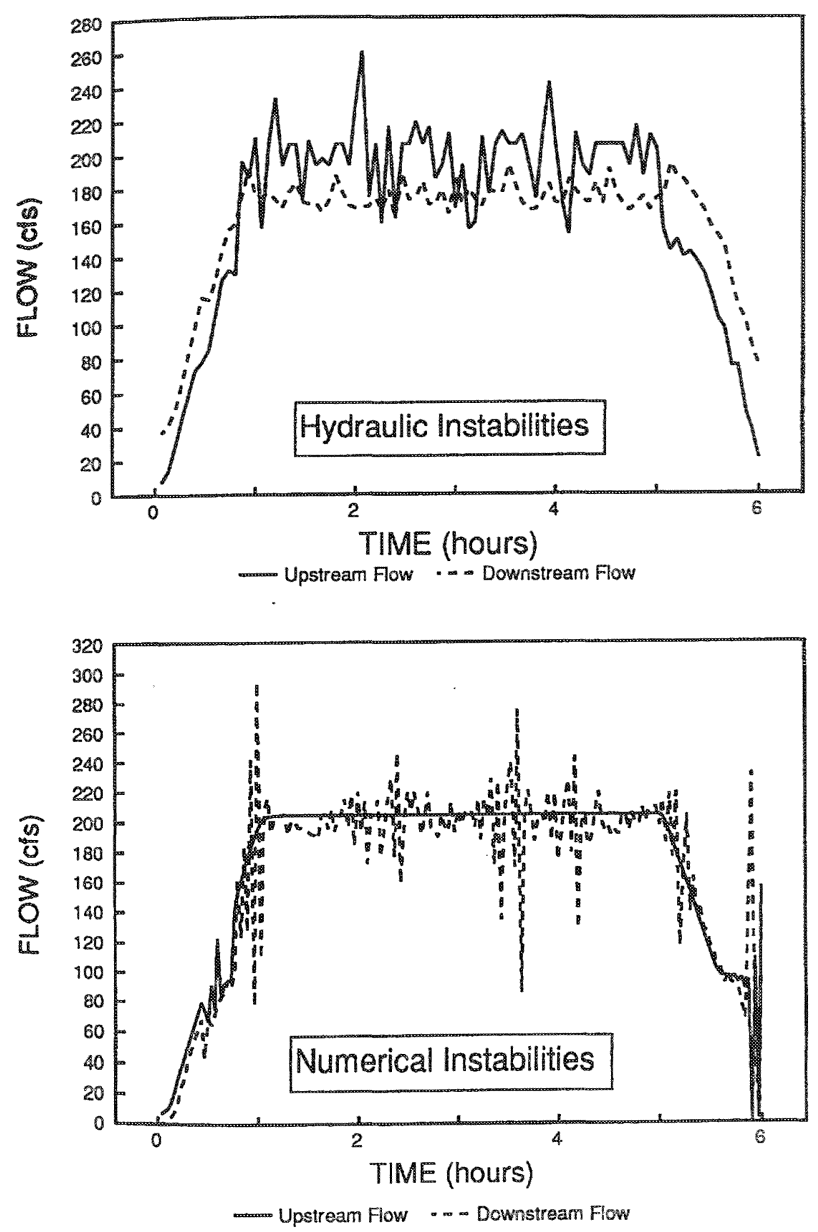

Figure 22.12 Computed instabilities. 


\subsection{Special Flow Conditions}

\subsubsection{Free Discharge Only}

For control gates found in sewer systems, the gate operation or the layout of the surrounding pipes and regulating chambers may be designed to ensure that free discharge occurs under the gate at all times, or at least under most normal operating conditions. One such instance would be when the pipe downstream of the gate is sufficiently steep to ensure that supercritical flow is maintained at all times. Another example would be when a vertical shaft is present downstream of the gate, as is the case with two of the radial gates in the Vancouver Sewerage Area. In these cases, downstream conditions have no influence on the gate discharge, and it would be incorrect to include the downstream conditions in the discharge computations.

By setting the variable DISCND $=2$, RGEXTRAN accounts for these conditions and assumes that free discharge occurs at the gate for most conditions. The new code also makes provision for the gate to become submerged under extreme conditions. The assumption is made that the gate is submerged when the downstream junction stage (Figure 22.4) exceeds the gate lip elevation:

where:

$$
D N I N V+H D>U P I N V+Y I N V R T+G O P E N
$$

DNINV = invert elevation downstream of the gate in $\mathrm{rn}[\mathrm{ft}]$,

$H D=$ flow depth downstream of the gate in $\mathrm{rn}[\mathrm{ft}]$,

UPINV = invert elevation upstream of the gate in $\mathrm{m}[\mathrm{ft}]$,

YINVRT = height of gate sill in $\mathrm{m}[\mathrm{ft}]$, and

GOPEN = vertical gate opening in $\mathrm{m}[\mathrm{ft}]$.

When the condition in the above equation is met, the gate discharge is computed by the generalized orifice equation, using the constant discharge coefficient. The influence of the downstream conditions is then accounted for by using the following definition for the head term:

$$
H=H_{1}-H_{2}
$$

where $H_{1}$ and $H_{2}=$ stage at the upstream and downstream nodes in $\mathrm{ft}(\mathrm{m})$.

When this submerged condition is no longer valid the program continues the simulation using only the free discharge algorithms as before and the downstream conditions are ignored in the discharge computations. It the free 
discharge assumption is valid, use of this option eliminates the submerged discharge computations and the test for determining the flow condition (shown in Figure 22.13), resulting in a significant decrease in the model run time.

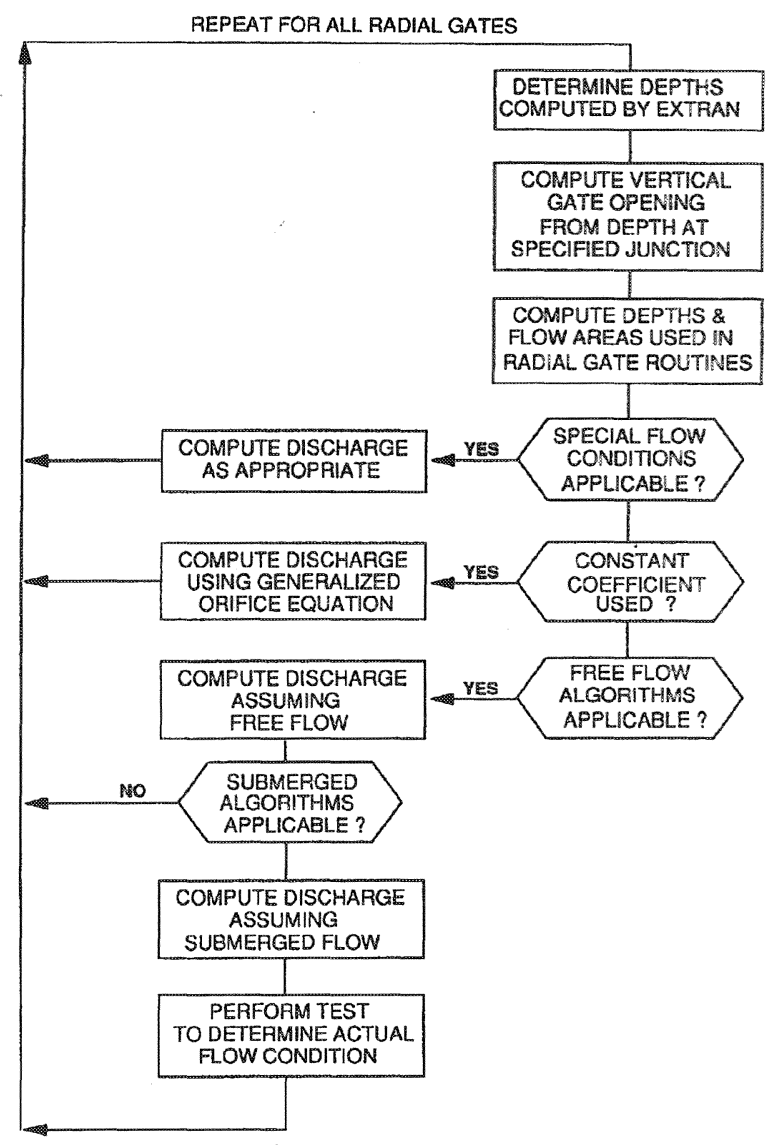

Figure 22.13 Radial gate logic.

\subsubsection{Use of Constant Discharge Coefficient}

The program allows for the specification of a constant discharge coefficient CDRG to be used in place of the BUREC algorithms. This is done by setting the parameter DISCND to 3 . A constant discharge coefficient has been used for sluice gates in UNSTDY (Book, 1980), and evidently produced satisfactory 
results. Constant discharge coefficients are also used in EXTRAN for weir and orifice diversions. While the BUREC algorithms are directly related to radial gates, use of a constant discharge coefficient may be used to model other gate types. This simplified approach may be less accurate than using the BUREC algorithms but will generally produce more stable results, under submerged flow conditions.

\subsubsection{DISCND Parameter}

The DISCND parameter has been included in the code to give the user more control over the radial gate computations. Use of this parameter may alleviate stability concerns and allow the code to simulate a wider range of conditions. Extensive testing was done to determine the effects of this parameter, and to provide general guidelines for optimum use of the program. The conclusions are summarized in Table 22.1 below.

Table 22.1 Use of the DISCND parameter.

\begin{tabular}{|c|c|c|c|}
\hline & Description & When to use? & When not to use? \\
\hline 0 & $\begin{array}{l}\text { Replaces radial gate link by } \\
\text { equivalent pipe. Constant } \\
\text { discharge coefficient is used. }\end{array}$ & $\begin{array}{l}\text { Submerged discharge only. } \\
\text { Surcharge conditions. } \\
\text { Steep slopes and steep } \\
\text { hydrographs. Should only } \\
\text { be used when stability } \\
\text { problems are encountered } \\
\text { with other options. }\end{array}$ & $\begin{array}{l}\text { Gate discharge is free. } \\
\text { Use of equivalent pipe } \\
\text { (increased volume) is not } \\
\text { acceptable. }\end{array}$ \\
\hline 1 & $\begin{array}{l}\text { Uses full BUREC algorithms } \\
\text { for radial gate discharge } \\
\text { coefficients. Performs test for } \\
\text { discharge condition (free or } \\
\text { submerged). Accounts for } \\
\text { gate sill, local energy losses, } \\
\text { and gate lip seal. }\end{array}$ & $\begin{array}{l}\text { For gradually varying flow } \\
\text { conditions and mild } \\
\text { channel slopes. } \\
\text { May be used effectively if } \\
\text { model is used as an } \\
\text { analysis tool (e.g. to } \\
\text { produce a rating curve or } \\
\text { to determine the discharge } \\
\text { condition for a range } \\
\text { of operating conditions) }\end{array}$ & $\begin{array}{l}\text { Rapidly varying storm } \\
\text { conditions where stability } \\
\text { is a concern. Details of } \\
\text { gate and regulating } \\
\text { chamber are not known } \\
\text { (i.e., gate sill, energy } \\
\text { losses, etc). Surcharge } \\
\text { conditions. }\end{array}$ \\
\hline 2 & $\begin{array}{l}\text { Uses BUREC free discharge } \\
\text { algorithms only. } \\
\text { Submergence criteria given } \\
\text { by sumergence equation. }\end{array}$ & $\begin{array}{l}\text { Radial gate discharge is } \\
\text { free at most times (i.e., } \\
\text { downstream conditions do } \\
\text { not influence gate } \\
\text { discharge). }\end{array}$ & $\begin{array}{l}\text { Gate discharge is } \\
\text { submerged. }\end{array}$ \\
\hline 3 & $\begin{array}{l}\text { Uses constant discharge } \\
\text { coefficient and generalized. } \\
\text { To represent other gate types }\end{array}$ & $\begin{array}{l}\text { Details of gate and } \\
\text { regulating chamber are not } \\
\text { known. To represent other } \\
\text { gate types (e.g. sluice } \\
\text { gate). }\end{array}$ & Stability is a concern. \\
\hline
\end{tabular}




\subsubsection{Logic Structure of Radial Gate Computations}

The computations at each time-step begin by identifying the depths, computed in the routing procedure, at the upstream and downstream junctions of each radial gate. The vertical gate opening is determined from conditions at the remote controlling node. The program then modifies the junction depths to account for the presence of a gate sill and any invert discontinuities between the junctions and the radial gate section (Figure 22.4), and computes the cross sectional flow areas upstream and downstream of the gate, based on the dimensions of the upstream and downstream conduits (which must be of rectangular cross-section). The program checks if any of the special flow conditions, discussed previously, are applicable. If so, the gate discharge is computed from the simplified equations. These special conditions refer to any condition to which the full BUREC discharge algorithms are not applicable. If the BUREC algorithms are applicable, the radial gate discharge is computed by the procedures previously described. The logic of the discharge computations is given by the flow chart in Figure 22.13. The computations for equivalent pipes are not done in the radial gate routines, but are performed together with the routing calculations.

\subsubsection{Model Calibration using Radial Gate Parameters}

Several parameters in the new radial gate code may be used in the calibration process. For gates where the equivalent pipe or constant discharge coefficient options are used, the discharge coefficient CDRG may be effectively used. For gates using the BUREC algorithms, the variables CFDA and CSDA are most suitable. If adequate data is available, more refinement on the gate computations is possible by varying UPTRNS and DNTRNS to account for head losses caused by structural changes in the gate intake and outflow sections shown in Figure 22.4. The relative sensitivity of these parameters and their effect on the gate computations is illustrated in Figure 22.14. For cases where the unrestricted discharge condition is important, RLRUFF may be used for refining the computations under these conditions. However, this parameter is relatively insensitive to change, as shown in Figure 22.14. If dynamic controls are present, it is important that the correct operation of the prototype gate be identified before applying the model, as this has far more influence on the model results than the parameters discussed above. 

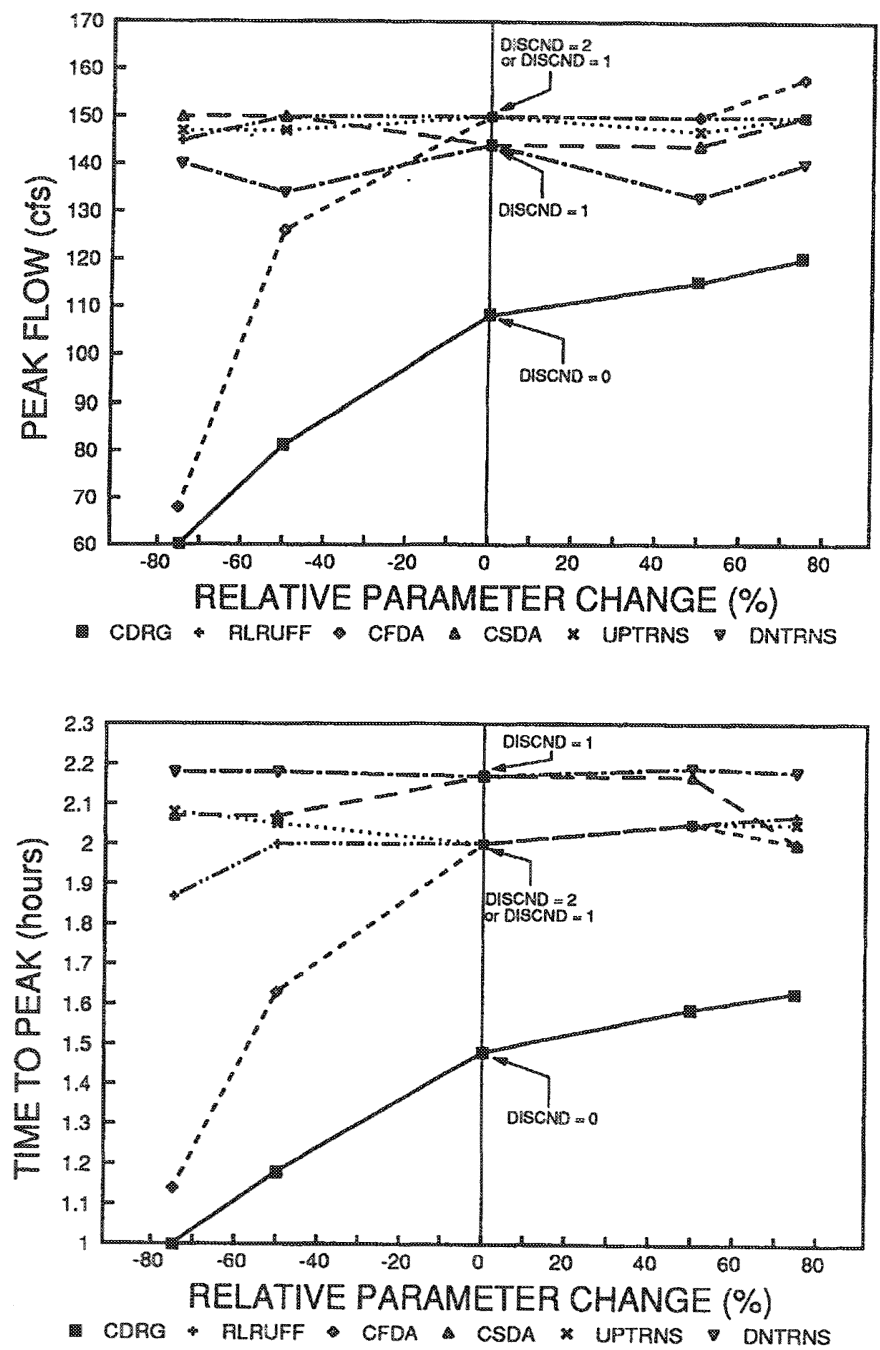

Figure 22.14 Radial gate routine, calibration.

\subsubsection{Output from Radial Gate Computations}

The output from radial gate computations is in the form of a time-history of the radial gate discharge and vertical gate opening, which is tabulated in the results file. As it may be desirable to consider optimization of the system by modifying the dynamic operation of these gates, the output of discharge and gate opening provides a valuable tool for this analysis. 


\subsection{Conclusions}

A new program, RGEXTRAN, was developed for complex combined sewer systems with dynamically-controlled radial gates (and other gate types). RGEXTRAN combines EXTRAN with new source code developed for general gates including radial gates. The new code uses special algorithms developed by the U. S. Bureau of Reclamation (BUREC) to compute the discharge coefficients for radial gates. The new gate code also simulates dynamic operation of the radial gates, when controlled by water levels at remote locations in the system (usually in the downstream interceptor). The code was extensively tested and verified for all possible unsteady flow conditions that may occur in the prototype sewer system, including: zero flow, surcharge, and flow reversal.

Verification of the code was deemed to be successful and the model results have been found to be consistent with computed theoretical results. Continuity is maintained at the radial gate locations at all times, except under unstable conditions. Under certain conditions, the radial gate computations may be susceptible to hydraulic instabilities. However, special steps can be taken to avoid these instabilities.

Probably the most successful part of this work was that it showed that additional code can be successfully written for special control structures and integrated into the overall EXTRAN model, using steady state equations. This gives the overall model more flexibility and utility and is more important than applying for example theory specific to radial gates. This work represents the first application of special real-time control structures in EXTRAN. Extensive testing showed that (given the small range of operation of these gates) exact geometry and other factors do not significantly affect the results. In practice a constant $C_{d}$ was more often used than the complex BUREC algorithms.

\section{Acknowledgments}

Financial support for this project was provided by the Greater Vancouver Regional District, Computational Hydraulics International, the Natural Science and Engineering Research Council of Canada, and the University of Guelph. RGEXTRAN was developed by Brett Young as part of the requirements for his MSc degree at the University of Guelph (Young, 1991), under Bill's "advisement". 


\section{References}

Book, D. E. 1980. A Dynamic Hydraulic Model for Simulation and Evaluation of Complex Drainage Systems. M.Sc. thesis, Colorado State University, Colorado. p. 54-57.

Bos, M.G. (Editor). 1978. Discharge Measurement Structures. 2nd Ed., Publication 20, Working Group on Small Hydraulic Structures. Inter. Inst. for Land Reclamation and Improvement (ILRI), Wageningen, Netherlands. p. 309-314.

Buyalski, C.P. 1983. Discharge Algorithms for Canal Radial Gates. Technical Report: RECERC-83-9. Bureau of Reclamation, U.S Department of the Interior.

Chadwick, A.J. and J.C. Morfett. 1986. Hydraulics in Civil Engineering. Allen \& Unwin. p. 406-410.

Chow, V.T. 1959. Open Channel Hydraulics. McGraw-Hill Book Company: New York. p. 507-512.

Franke, P.G. and F. Valentine. 1969. The Determination of Discharge below Gates in the case of Variable Tailwater Conditions. Journal of Hydraulic Research 17 (4). p. 433-447.

Gill, M.A. 1982. Discharge Characteristics of Radial Gates. International Water Power and Dam Construction 8 (3). p. 39-42.

Hamilton, D.L. 1985. Microcomputer Simulation of Canal Operation. M.Sc. Thesis, U of California, Davis. p. 26-28.

Henderson, F.M. 1966. Open Channel Row. MacMillan Publishing Co. Inc.: New York. p. 202-210.

Huber W.C. and R.E. Dickinson. 1988. Storm Water Management Model User's Manual, Version 4. Environmental Protection Agency: Athens, Georgia. p. 136139.

Isaacs L.T. 1977. Numerical Solution for Flow under Sluice Gates. Journal of the Hydraulics Division. American Society of Civil Engineers 103 (HY5). p. 473-481.

Larock, B.E. 1970. A Theory for Free Outflow beneath Radial Gates. J. of the Fluid Mechanics 41 (4):851-864.

Lewin, J. 1980. Hydraulic Gates. Instn of Water Engineers and Scientists Journal 34 (3). p. 237-255.

Metzler, D.E. 1948. A Model Study of Tainter-Gate Operation. M.Sc. Thesis, U of Iowa, Iowa City.

Ranntz, S.E. 1982. Measurement and Computation of Streamflow Vol. 2: Computation of Discharge. Geological Survey Water-Supply Paper 2175. U.S. Department of the Interior: Washington. p. 499-508.

Roesner, L.A. , J.A. Aldrich, and R.E. Dickinson. 1989. Storm Water Management Model User's Manual Version 4: EXTRAN Addendum. USEPA: Athens, Georgia.

Toch, A.T. 1953. Discharge Characteristics of Tainter Gates. ASCE Hyd. Div. 79 (295). p.1-20.

Young, B. C. 1991. Dynamic modelling of combined sewer systems with radial gates and siphon weirs. MSc dissertation. Univ. Of Guelph, 1991. ca 120 pp excl. appendices on disk. 